Research Article

\title{
Phased Array Synthesis Using Modified Particle Swarm Optimization
}

\author{
M. A. Zaman *, S. A. Mamun, Md. Gaffar, S. M.Choudhury, Md. M. Alam, and Md. Abdul Matin. \\ Department of Electrical and Electronic Engineering, Bangladesh University of Engineering and Technology, Dhaka - 1000, Bangladesh.
}

Received 2 September 2010; Revised 4 October 2010; Accepted 25 January 2011

\begin{abstract}
In this paper, a linear phased array is synthesized to produce a desired far field radiation pattern with a constraint on sidelobe level and beamwidth. The amplitude of the excitation current of each individual array element is optimized to give desired sidelobe level and beamwidth. A modified particle swarm optimization (PSO) algorithm with a novel inertial weight variation function and modified stochastic variables is used here. The performance of the modified PSO is compared with standard PSO in terms of amount of iterations required to get desired fitness value and convergence rate. Using optimized excitation amplitudes, the far field radiation pattern of the phased array is analyzed to verify whether the design criterions are satisfied.
\end{abstract}

Keywords: Antenna array, particle swarm optimization, phased array.

\section{Introduction}

A phased array antenna is composed of groups of individual radiating elements, which are distributed and oriented in a linear or two dimensional spatial configuration [1]. The amplitude and phase excitation of each element can be individually controlled to generate a radiation pattern of any desired shape. The angular position of the beam in space can be controlled electronically by adjusting the phase of the excitation currents of each individual element. Thus, beam scanning can be accomplished without any mechanical motion of the radiating elements.

Phased array antennas are widely used in Radar systems because of their rapid and accurate beam scanning capabilities [1]. For practical Radar applications, it is required that the phased array produces low sidelobe levels (SLL) with narrow beamwidth [2], [3]. The amplitude excitations of the array elements are selected to synthesize the desired pattem. The binomial method and the DolphTschebyscheff method are often used to synthesize radiation pattem with low SLL [2], [4]. But these methods do not provide the designer with the flexibility to set multiple design goals such as SLL, beamwidth and null control. So, it is often necessary to use an optimization algorithm to find the amplitude excitations of the array elements which give the desired results.

Evolutionary optimization algorithms often outperform classical optimizations algorithms and give more flexible solutions than analytic approaches. Particle Swarm Optimization (PSO) is one such evolutionary algorithm. It is based on the movement and intelligence of swarms [5]. Like the widely used Genetic Algorithm (GA), PSO has been found to be effective in many electromagnetic problems [6]. PSO can be used to optimize the excitation amplitudes of the array elements to synthesize a predetermined pattern [7], [8].

The time limiting step of any optimization process is the evaluation of the fitness function. The evaluation of fitness function in array synthesis requires computation of radiation pattem, SLL and beamwidth. Due to the high computational efforts required, the optimization process is often slow. As the fitness function can not be altered, it is highly desired that the optimization algorithm requires low number of iterations to converge. The standard PSO and some of its improved versions are moderately fast converging, which makes them suitable for array synthes is.

Some work has been done on array synthesis using different versions of PSO [7]-[9]. But none of these methods significantly increase the convergence rate. In this paper, some modifications of the standard PSO are introduced which increase the convergence rate of the optimization algorithm and reduce computational time. The modified PSO is used to calculate the excitation amplitudes of the array elements. The performance of the modified PSO is compared with standard PSO to verify the improvement of convergence rate. Finally, the far field radiation pattem of the synthesized array is analyzed.

\section{Phased Array Pattern Formulation}

The geometry of a linear phased array along with the three dimensional polar coordinate system is shown in Fig. 1. The array contains $2 M$ elements which are symmetrically placed on positive and negative side of the $\mathrm{z}$ axis. The excitation amplitude distribution is also assumed to symmetric about 
the origin. The far field radiation pattem of the phased array is given by [3], [4]:

$$
F F(\theta, \phi)=E P(\theta) \cdot A F(\theta, \phi)
$$

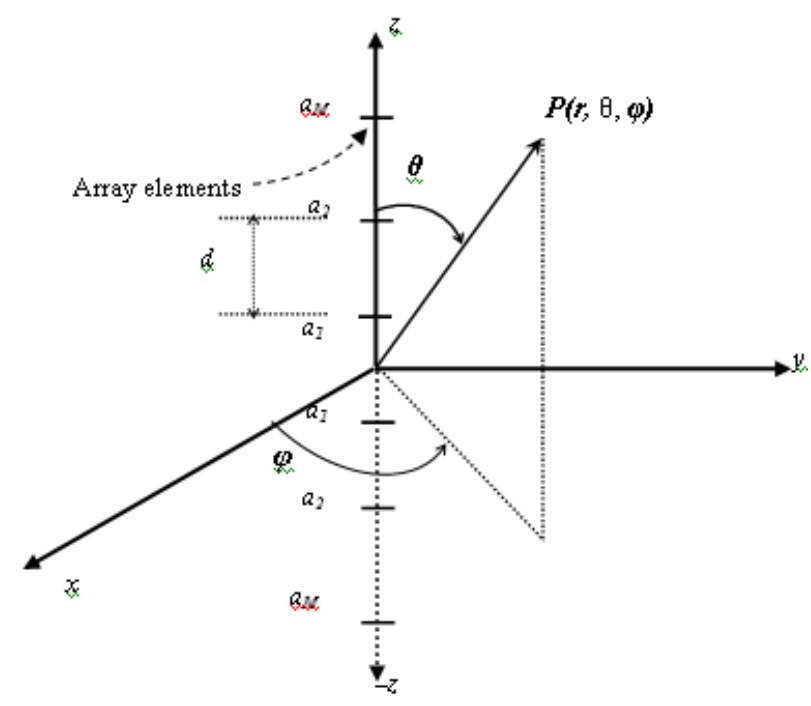

Fig. 1. Geometry of a $2 M$ element linear phased array.

Here, $A F(\theta, \phi)$ is the array factor and $E P(\theta)$ is the radiation pattem of the array elements. For practical phased arrays using aperture elements, the element pattem is often approximated by [1], [9]:

$$
E P(\theta)=\sqrt{\cos ^{n}(\theta)}
$$

Where, $n=0$ represents an ideal is otropic source and $n>$ 0 represents directive sources. In this paper, $n$ is taken to be 1.2 and all the elements are assumed to be identical.

For a linear array with $2 M$ elements placed symmetrically along the $\mathrm{z}$ axis, the array factor is given by [3]:

$$
A F(\theta, \phi)=A F(\theta)=2 \sum_{n=1}^{M} a_{n} \cos \left[\frac{(2 n-1)}{2} \psi\right]
$$

Here,

$\psi=k d \sin \theta+\beta$,

$\beta=-k d \sin \theta_{0}=$ phase excitation,

$\theta_{0}=$ desired angular position of the main beam,

$a_{n}=$ amplitude excitation of the $n^{\text {th }}$ array element,

$k=$ wave number $=2 \pi / \lambda$ where, $\lambda=$ wave length, and

$d=$ separation between two consecutive array element.

For numeric simulation, $d$ is taken to be $0.5 \lambda$.

The value of the phase excitation can be electronically controlled to perform beam scanning. In this paper the values of the excitation amplitudes, $a_{n}$, are optimized to generate a desired radiation pattern with low SLL. In this paper a 44 element phased array is designed. For uniform excitation, the beamwidth of such an array is approximately $8^{\circ}$ to $9^{\circ}$ and SLL level about $-18 \mathrm{~dB}$. The design goal is that the phased array will have SLL less than $-47 \mathrm{~dB}$ and beamwidth of approximately $9^{\circ}$. These values are not chosen completely arbitrarily. For a 44 element array, the lowest SLL level achievable by excitation optimization is about -45 $\mathrm{dB}$. And by minimizing SLL, the beamwidth can not be lowered more than $9^{\circ}$. However, a phased array for any arbitrary SLL and beamwidth can be designed using the same procedure used here by increasing or decreasing the number of array elements. So, the normalized desired pattem is defined as:

$$
F F_{\text {desired }}(\theta)=\left\{\begin{array}{ll}
0 d B, & -4.5^{\circ} \leq \theta \leq 4.5^{\circ} \\
-47 d B, & \text { otherwise }
\end{array}\right. \text {. }
$$

The radiation of the array for uniform excitation $\left(a_{n}=\right.$ constant $=1$ ) along with desired radiation pattem is shown in Fig. 2. It can be seen that, uniform excitation does not satisfy design requirements. So, optimization is necessary. The fitness function (cost function) is defined as the sum of the squares of the excess far field magnitude above the desired pattern. So,

Fitness $=\left\{\begin{array}{lc}\sum_{\theta=180^{\circ}}^{180^{\circ}}\left[F F(\theta)-F F_{\text {desired }}(\theta)\right]^{2}, & F F(\theta)>F F_{\text {desired }}(\theta) \\ 0, & \text { otherwise }\end{array}\right.$

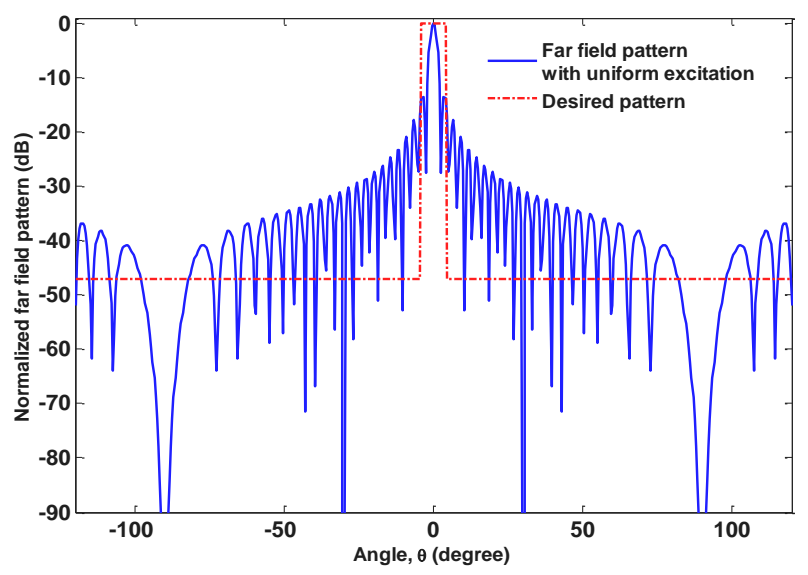

Fig. 2. Far field radiation pattern of the array with uniform excitation (blue) and desired array pattern (red) with $\theta=0^{\circ}$.

This type of fitness function definition is widely used for antenna array synthesis problems because it penalizes sidelobe levels above the desired envelope, while neither penalty nor reward is given for sidelobes below the specifications [7]. The target of the optimization process is to find the values of $a_{n}$, so that the fitness function is minimized and the achieved radiation pattem matches closely with the desired pattem.

\section{Modified Particle Swarm Optimization}

Particle swarm optimization is a high performance robust optimizer. It is an evolutionary algorithm which originated 
from the studies of bird flocking, fish schooling and bee swarms [5]. The swarm intelligence based PSO requires less computational book keeping than other evolutionary algorithms like genetic algorithms .

To solve an optimization problem of $N$ variables with PSO, a collection or swarm of particles is defined. Each particle is initially assigned a random position in the $N$ the dimensional problem hyperspace. The total number of particles is known as the population size. Each of these particle positions represents a possible solution of the optimization problem. Each particle is assigned a scalar fitness or cost value based on its position on the problem hyperspace. Thus, the fitness value indicates how well the particle solves the problem. The motion of the particles through the problem space is based on some deterministic and stochastic position update rules. As the particles travel the problem space, each particle remembers its own pers onal best position (which resulted in the best fitness value) that it has ever found. Each particle also knows the overall best position found by any particle in the swarm, called global best. The motion of each particle is affected by its personal best position and the global best position of the swarm. Over successive iterations, the personal and global best positions pull the particles near them. After sufficient number of iterations, all the particles converge and settle down near a good solution, thus completing the optimization process.

The position of a particle of the swarm can be represented as a vector, $\boldsymbol{x}=\left(x_{1}, x_{2}, \ldots \ldots \ldots x_{M}\right)$. The position of each particle is updated in each iteration step using the following equation:

$$
x_{n}(t+1)=x_{n}(t)+v_{n}(t) \cdot \Delta t
$$

Here, $t$ denotes iteration step, $\Delta t$ represent time step (which is taken to be unity), $x_{n}$ denotes the coordinate of the particle in $n^{\text {th }}$ dimension and $v_{n}$ denotes the velocity of the particle in the $n^{\text {th }}$ dimension. In standard PSO, the velocity at each iteration step is calculated from:

$$
\begin{aligned}
& v_{n}(t+1)=w v_{n}(t)+c_{1} r_{1}\left\{x_{p b, n}-x_{n}(t)\right\}+ \\
& +c_{2} r_{2}\left\{x_{g b, n}-x_{n}(t)\right\}
\end{aligned}
$$

Here, $r_{1}$ and $r_{2}$ are two random numbers between 0 and $1, c_{1}$ and $c_{2}$ are acceleration constants, $w$ is the inertial weight, $x_{p b, n}$ is the coordinate of the personal best position of the particle in $n^{\text {th }}$ dimension and $x_{g b, n}$ is the coordinate of the global best position of the swarm in the $n^{\text {th }}$ dimension. The value of both acceleration constants $c_{1}$ and $c_{2}$ are taken to be 2, which are commonly used values [6], [8]. The two random variables are used to simulate the slight unpredictable component of natural swarms. The inertial weight, $w$, determines to what extent the particle remains along its original course and is not affected by the pull of personal best and global best. In most cases, it has been found that linear decrease of $w$ from $w_{\max }=0.9$ to $w_{\min }=0.4$ gives good results [6]. If $t_{\max }$ is the maximum number of iterations, then, $w$ is expressed as:

$$
w(t)=w_{\max }-\frac{w_{\max }-w_{\min }}{t_{\max }} t
$$

In this paper, rather than using a linear variation, a novel Gaussian variation of $w$ is used. The modified expression is:

$$
w(t)=\left(w_{\max }-w_{\min }\right) \exp \left\{-\left(\frac{z t}{t_{\max }}\right)^{2}\right\}+w_{\min }
$$

Here, $z$ is a constant whose value is taken to be 2.2. This modified inertial weight variation function is shown in Fig. 3. The function has the same initial and final values as the linear function, but it is characterized by a sharper rate of decrease. A small value of $w$ encourages local exploitation whereas a larger value of $w$ encourages global exploration [6]. So, it is expected that the sharper fall of $w$ will increase the rate of convergence without sufficiently reducing global search.

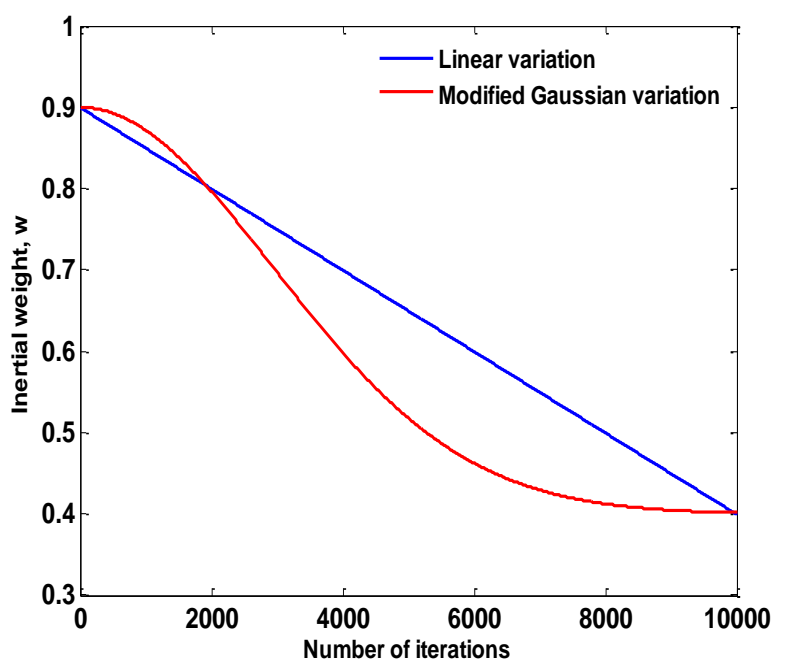

Fig. 3. Standard and modified inertial weight variation.

The two random variables $r_{1}$ and $r_{2}$ used in (5) affect the pull of personal best and global best. As the variables are independent of each other, they do not represent natural swarm behavior correctly. This often leads to slow convergence. So, relating $r_{1}$ and $r_{2}$ in such a way that both of them are not large or small at the same time is expected to improve the convergence [9]. Using a simple linear relationship between the variables, the velocity update equation can be modified. This modified equation can be expressed as:

$$
\begin{aligned}
& v_{n}(t+1)=w(t) v_{n}(t)+c_{1} r_{1}\left\{x_{p b, n}-x_{n}(t)\right\}+ \\
& +c_{2}\left(1-r_{1}\right)\left\{x_{g b, n}-x_{n}(t)\right\}
\end{aligned}
$$

The modified PSO used here consists of this modified velocity update equation along with modified inertial weight variation function given by (7).

\section{Numerical Simulation Results}

For numerical computation, a 44 element $(2 M=44)$ phased array is simulated. Population size is selected to be 25 . Maximum iteration number, $t_{\max }$, is set to 10000 . As the 
amplitude excitation of the array elements are assumed to be symmetrically distributed; only the amplitudes of $M$ elements located at the positive side of $\mathrm{z}$ axis are optimized. The excitation of the elements located at the negative side of the $\mathrm{z}$ axis are assigned values same as corresponding positive side elements.

Fig. 4 shows the comparis on of fitness function values for standard PSO and modified PSO. The same set of initial random values is used for both methods to ensure proper comparison. It is clear that the average fitness and best fitness both reach desired low values faster in the case of modified PSO.

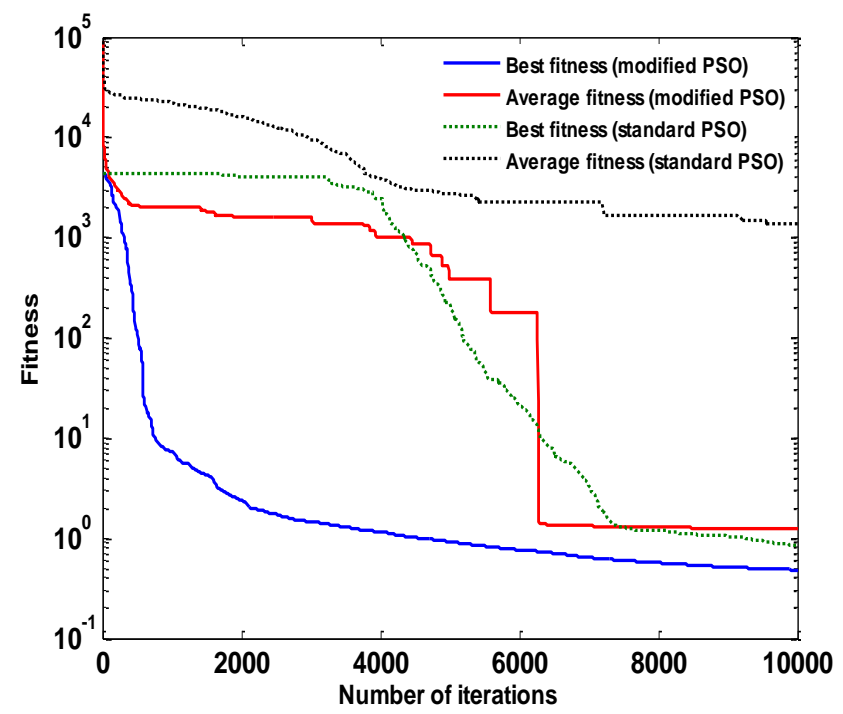

Fig. 4. Comparison of fitness function values for standard and modified PSO.

To verify the superior performance of the modified PSO over classical PSO, both algorithms are simulated multiple times. To make sure that the comparis on is unbiased, the initial random position and random velocity of the particles are taken the same for both classical PSO and modified PSO. Two versions of each algorithm are tested. In the first version, the optimization process is assumed to be complete when the average fitness values falls below a certain threshold level. For the second version, the optimization process is assumed to be complete when the best fitness value falls below a threshold level. The threshold level is taken to be 10 for both cases. Also, maximum iteration number is limited to 10000 for both cases. The simulation results are shown in Tab. 1 . The results show that, modified PSO outperforms classical PSO for both versions. Modified PSO with best fitness threshold is found to be the fastest algorithm. Although, the average fitness threshold stop algorithm takes longer time to simulate, it ensures that the optimization procedure is not stopped prematurely. It is noted that the average fitness threshold based stop for classical PSO does not reach the break condition within the defined maximu miteration number.

Table1. Comparison of the performance of classical PSO and modified PSO.

\begin{tabular}{|c|c|c|c|c|c|c|c|c|}
\hline \multirow[t]{2}{*}{ 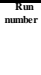 } & \multicolumn{2}{|c|}{ Avg, fingess thres hold break } & \multicolumn{2}{|c|}{ Best fithess Atres hold break } & \multicolumn{2}{|c|}{ Avg. finess tireshold break } & \multicolumn{2}{|c|}{ Best ffithess thres hold break } \\
\hline & 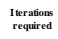 & $\begin{array}{c}\text { Simulation } \\
\text { time (sec) }\end{array}$ & 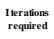 & $\begin{array}{c}\text { Simulation } \\
\text { time (sec) }\end{array}$ & $\begin{array}{l}\text { Itera aions } \\
\text { required }\end{array}$ & $\begin{array}{c}\text { Sinultion } \\
\text { tine (sec) }\end{array}$ & 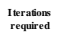 & $\begin{array}{l}\text { Simulation } \\
\text { fine sec }\end{array}$ \\
\hline$\frac{1}{2}$ & 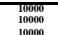 & 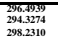 & $\begin{array}{l}62919 \\
61619 \\
61049\end{array}$ & 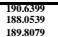 & 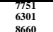 & 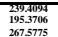 & $\begin{array}{l}16069 \\
1179 \\
13909\end{array}$ & 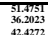 \\
\hline${ }_{5}^{4}$ & 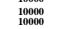 & 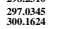 & 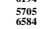 & 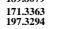 & $\begin{array}{l}2725 \\
7385 \\
9859\end{array}$ & 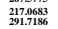 & $\begin{array}{l}2601 \\
2007 \\
2007\end{array}$ & 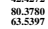 \\
\hline
\end{tabular}

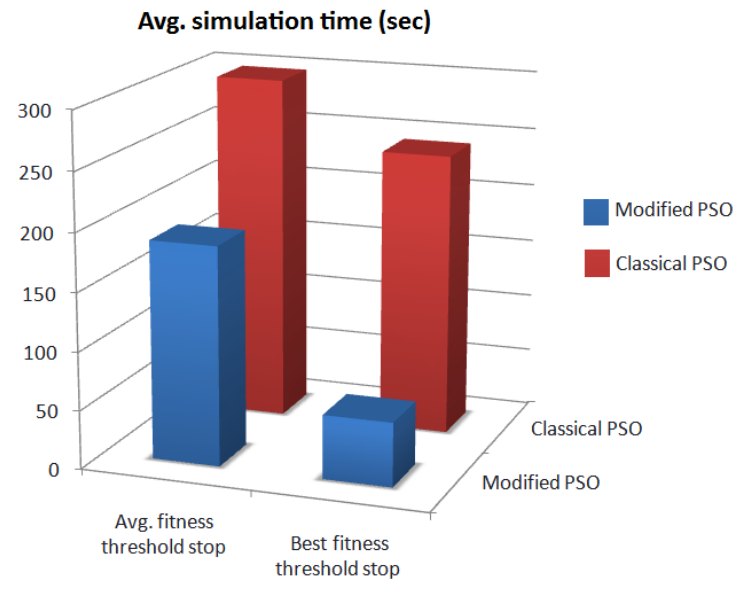

Fig. 5. Comparison of average simulation time classicalPSO and modified PSO.

The simulations are run on a computer with processor: Intel Core2 T5600 1.83GHz, AND RAM: 2.00 GB (DDR2). The simulation times are expected to vary with change in hardware, but the comparative performance of the two algorithms will be unchanged.

In Fig. 6, the values of a particular solution $\left(11^{\text {th }}\right.$ excitation amplitude) are shown as a function of number of iterations when using standard PSO. The same plot is shown in Fig. 7 when modified PSO is used. When the graph becomes horizontal, it indicates convergence. It is clear that the solution converges faster for modified PSO.

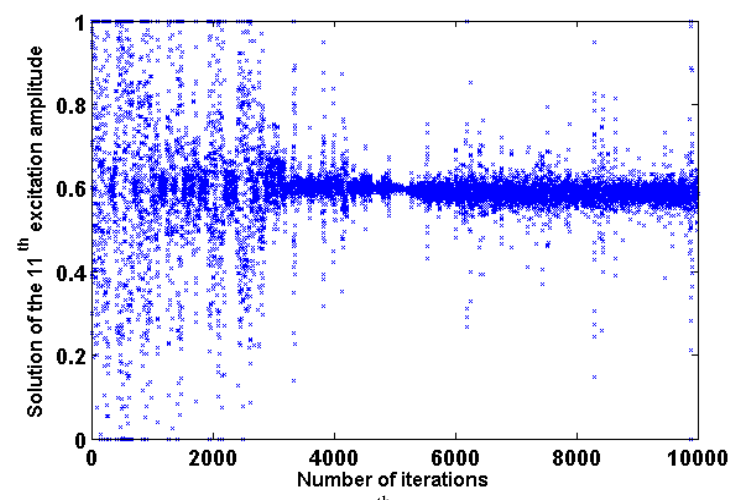
PSO

Fig. 6. Convergence of the $11^{\text {th }}$ excitation amplitude using standard

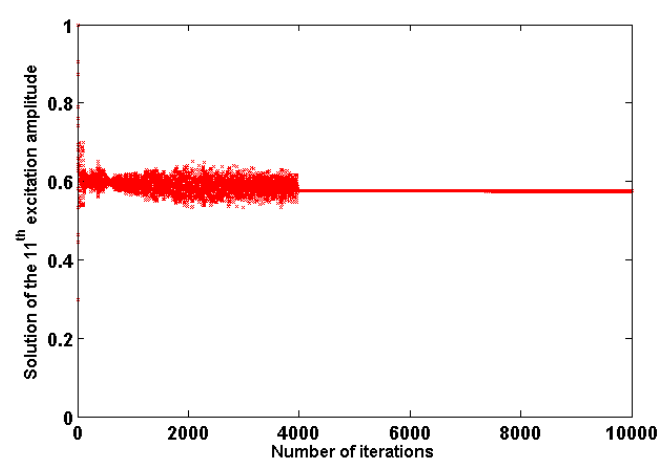

Fig. 7. Convergence of the $11^{\text {th }}$ excitation amplitude using modified PSO.

For simulation, the solution space is limited in each dimension to the range $x_{\min }=0$ to $x_{\max }=1$. To handle the 
situation where particles drift outside the solution space, absorbing wall boundary condition is used [6]. In this method, if the position coordinate of a particle position exceeds the limits in any dimension, it is set to the nearest boundary value $\left(x_{\max }\right.$ or $\left.x_{\text {min }}\right)$. Similarly, maximum velocity of a particle in any dimension is limited to the range -1 to +1 .

The optimized excitation amplitude gained from modified PSO is shown in Fig. 8. The 22 elements located at negative $\mathrm{z}$ axis are labeled sequentially from -22 to -1 and the 22 elements located at the positive side are labeled from 1 to 22. The resulting far field pattern is shown in Fig. 9. It is seen that optimized pattem matches very closely with the desired pattern. The best fitness value after 10000 iterations was 0.2670 , which is very close to the desired value of zero.

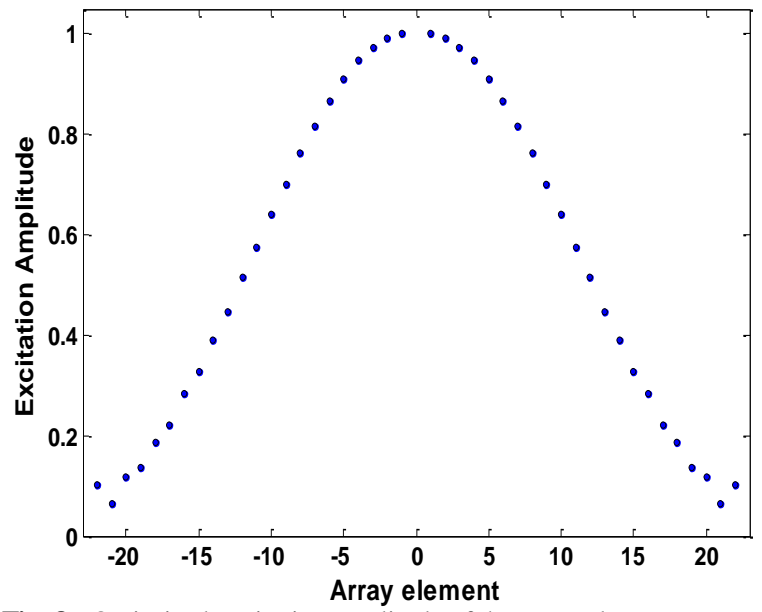

Fig. 8. Optimized excitation amplitude of the array elements.

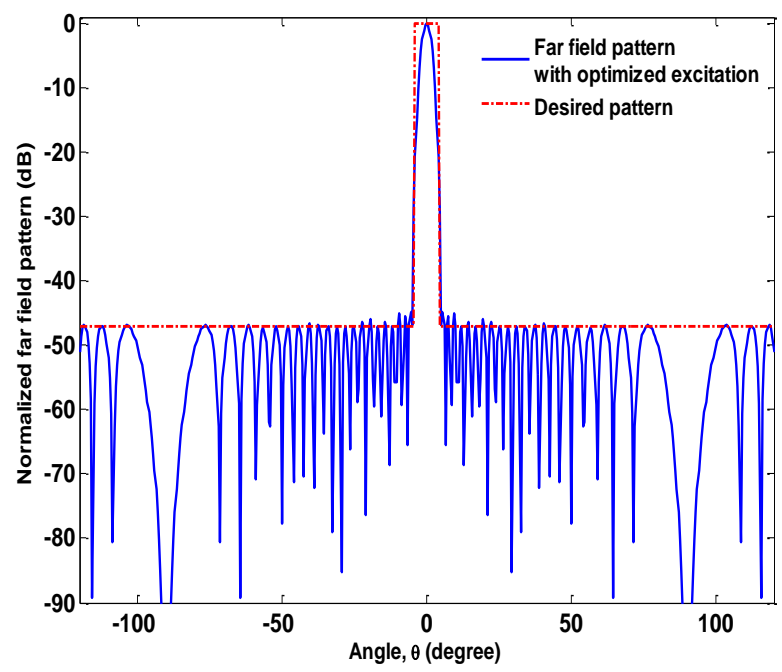

Fig. 9. Optimized far field radiation pattern $\left(\theta_{0}=0^{\circ}\right)$.

Fig. 10 shows the beam scanning operation of the phased array. When the phase excitation corresponding to $\theta_{0}=0^{\circ}$ is used, the main beam is located at $\theta_{0}=0^{\circ}$. When the excitation is changed to a value corresponding to $\theta_{0}=45^{\circ}$, it is found that the main beam shifts to $\pm 45^{\circ}$ accordingly. Fig. 11 shows the three dimensional far field radiation pattern of the phased array for $\theta_{0}=0^{\circ}$. It can be seen that the pattem is independent of $\varphi$ values, which is expected for a linear array along $\mathrm{z}$ axis.

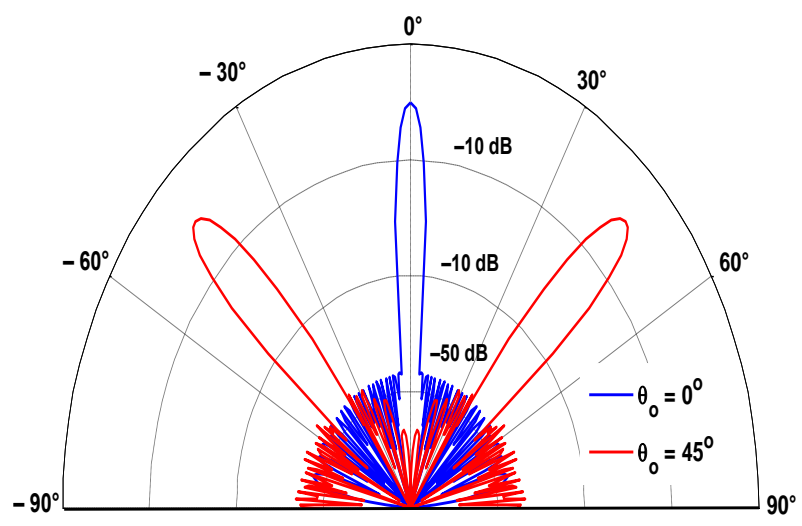

Fig. 10. Beam scanning operation of the optimized phased array with $\theta_{0}$ $=0^{\circ}$ (blue) and $\theta_{0}=45^{\circ}$ (red).

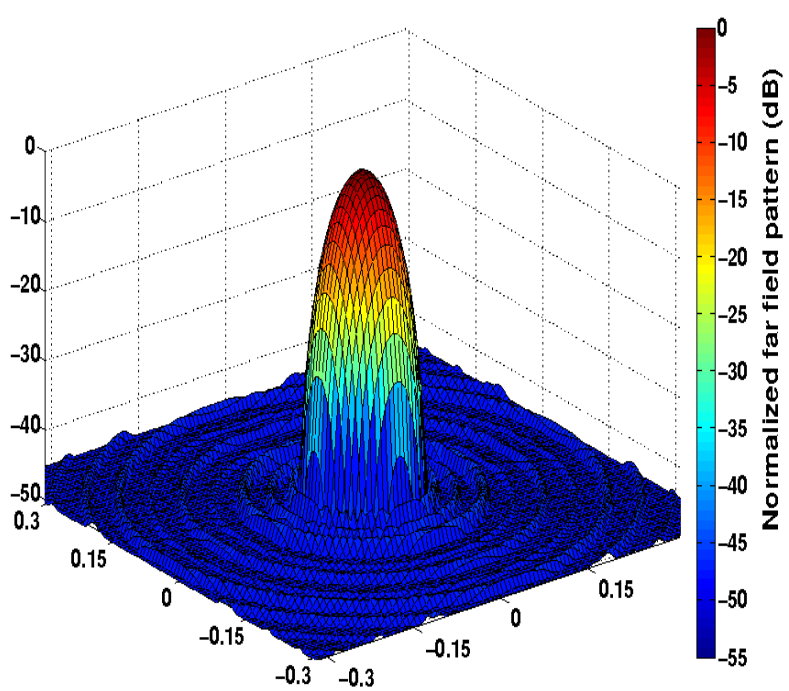

Fig. 11. Three dimensional far field radiation pattern of the optimized array for $\theta_{0}=0^{\circ}$.

A similar optimization procedure can be implemented to optimize arrays of Cassegrain reflectors used in radio astronomy. In that case, the individual element pattem must be modified accordingly [10].

\section{Conclusion}

A linear phased array is optimized and simulated in this paper. A modified PSO algorithm is used to optimize the amplitude excitation of the array elements to produce a desired far field pattern with low SLL and a specific beamwidth. It has been shown that the modified PSO outperforms standard PSO for this optimization problem. The simulation results show that the optimized phased array meets all the design requirements. 


\section{References}

1. R. C. Hansen, Antenna Engineering Handbook, Chapter 20: Phased Arrays, J. L. Volakis (Ed.), $4^{\text {th }}$ edition, McGraw-Hill (2007).

2. Balanis, Antenna Theory Analysis and Design, $3^{\text {rd }}$ edition, John Wiley \& Sons (2005).

3. Balanis (Ed.), Modern Antenna Handbook. John Wiley \& Sons, 2008.

4. J. D. Kraus, R. J. Marhefka, and A. S. Khan, Antennas for all applications, $3^{\text {rd }}$ edition, T ata McGraw-Hill (2007).

5. R. Eberhart, J. Kennedy, "A new optimizer using particle swarm theory ", $16^{\text {th }}$ International Symposium on Micro Machine and Human Science, pp. 39-43 (1995).

6. J. Robinson, Y. Rahmat-Samii, "Particle swarm optimization in elect romagnetics", IEEE T rans. Ant ennas Propagat., Vol. 52, no. 2, pp. 397-407 (Feb. 2004).

7. W. Boeringer and D. H. Werner, "Particle swarm optimization versus genetic algorithm for phased array synthesis", IEEE
Trans. Antennas Propagat., Vol. 52, no. 3,pp. 771-779 (March 2004)

8. T. B. Chen, Y. B. Chen, Y. C. Jiao, and F.S. Zhang, "Synthesis of antenna array using particle swarm optimization", AsiaPacific Microwave Conference Proceedings, Vol. 3, pp. 4-7 (Dec. 2005).

9. W. T. Li, S. F. Liu, and X. W. Shi, "Application of improved particle swarm optimization in antenna array pattern synthesis," Global Symposium on Millimeter Waves, pp.119-122 (April 2008).

10. M. A. Zaman, S. M. Choudhury, M. Gaffar, M. A. Matin, "Modeling the illumination function of a cassegrain reflector for a corrugated horn feed and calculation of the far field pattern," Loughborough Antennas \& Propagation Conference, LAPC, pp. 101-104, (Nov. 2009). 